
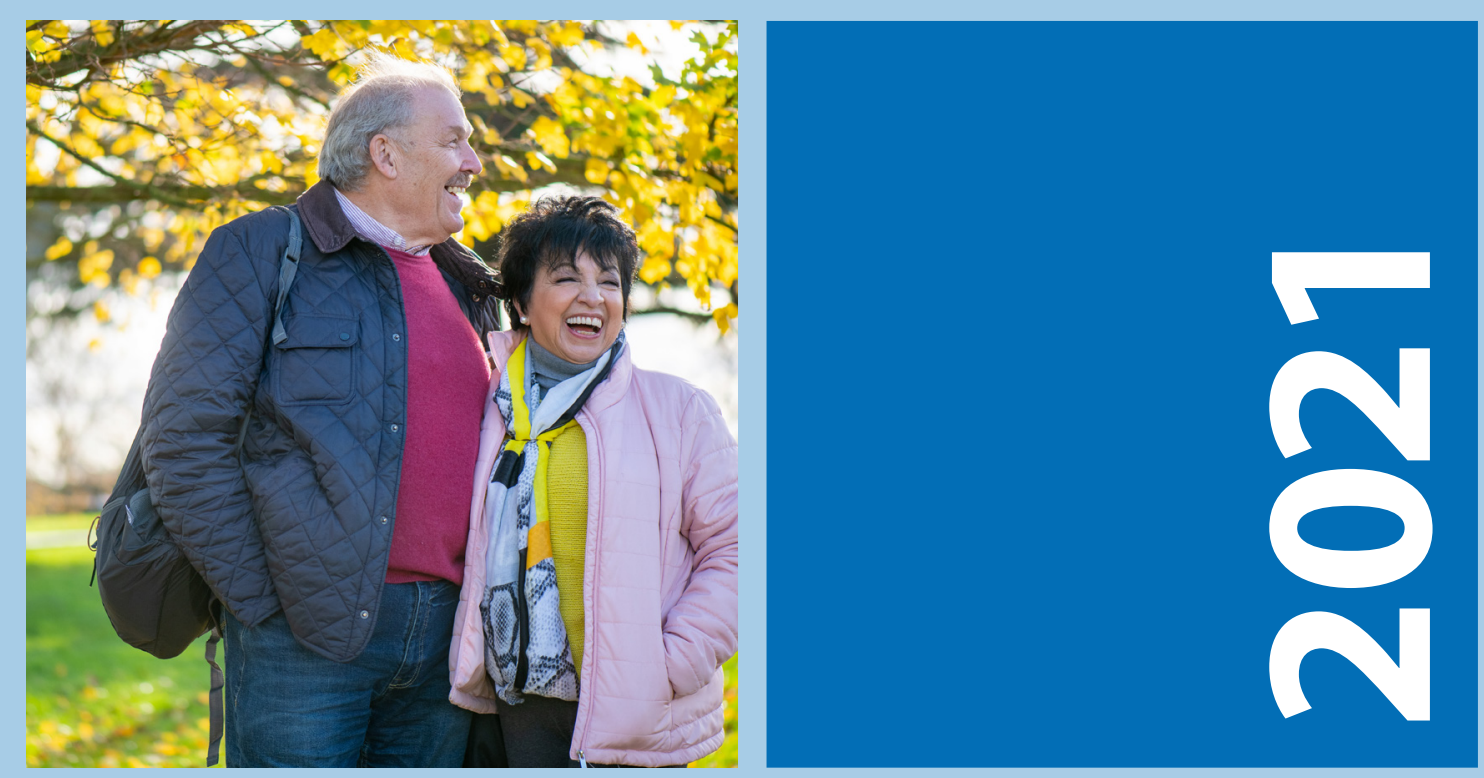

Preparing for COVID-19 vaccination: important learnings from the Irish Longitudinal Study on Ageing (TILDA)

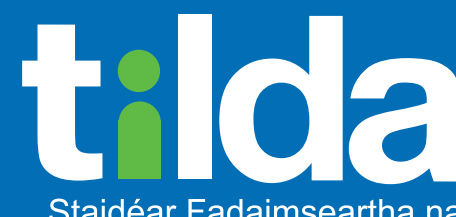

Staidéar Fadaimseartha na hÉireann um Dhul in Aois

The Irish Longitudinal Study on Ageing 


\section{Preparing for COVID-19 vaccination: important learnings from the Irish Longitudinal Study on Ageing (TILDA)}

Céline De Looze, Mark Ward, Cillian McDowell, Nollaig Bourke \& Rose Anne Kenny

On behalf of the TILDA team

January 2021 
Copyright $@$ The Irish Longitudinal Study on Ageing 2021

The Irish Longitudinal Study on Ageing

Trinity College Dublin

Dublin 2

Tel: +35318962509

Email: tilda@tcd.ie

Website: www.tilda.ie

https://www.doi.org/10.38018/TildaRe.2021-00 


\section{Acknowledgements}

We would like to acknowledge the vision and commitment of our study funders, the Department of Health, the Health Research Board, The Atlantic Philanthropies, and Irish Life plc. We would like to state that any views expressed in this report are not necessarily those of the Department of Health or of the Minister for Health. We would also like to thank the TILDA participants without whom this research would not be possible. 


\section{Table of Contents}

Introduction.

Influenza vaccination prevalence among TILDA participants in 2016-2019

2

Mark Ward

The effect of prolonged physical activity on vaccine efficacy

Nollaig Bourke

Levels of physical activity among TILDA participants who got the influenza vaccine between 2016 and 2019

Cillian McDowell

TILDA participants' response to government's communication during the COVID-19 pandemic

Mark Ward

Conclusion

References 


\section{Introduction}

- This report brings together important information on vaccinations among adults aged 60 years and older learned from the Irish Longitudinal Study on Ageing (TILDA), which can inform the rollout of COVID-19 vaccinations.

- The report describes:

i. the prevalence of influenza vaccination among TILDA participants between 2016 and 2019;

ii. (scientific evidence for the effect of prolonged physical activity on vaccine efficacy;

iii. the levels of physical activity among TILDA participants who got the influenza vaccine; and

iv. the news sources used during the COVID-19 pandemic and levels of trust in these sources among TILDA participants. 


\section{Influenza vaccination prevalence among TILDA participants in 2016-2019}

\section{Mark Ward}

The data reported in this section was collected by TILDA during the fifth wave of data collection in 2018/19.

Data on patterns of uptake of the influenza vaccine can provide useful information on likely patterns of vaccine uptake among community-dwelling adults aged 60 years and older in Ireland. In the fifth wave of data collected by TILDA in 2018/19, we asked if participants had received the influenza vaccine in the twoyears prior to their survey interview. For those who had received the influenza vaccination, we also asked who provided the vaccination (for example General Practitioner, Pharmacist etc).

- $59 \%$ of adults aged 60 years and older in Ireland received an influenza vaccination between 2016 and 2019.

- There was similar level of vaccination among men and women.

- As shown in Figure 1, the rate of influenza vaccination was significantly higher among the over 70 s: $76 \%$ availed of the vaccine compared to $41 \%$ of adults aged $60-69$ years.

Figure 1. Percentage of adults aged 60-69 and over 70 years who received the influenza vaccine in the two years prior to $2018 / 19$

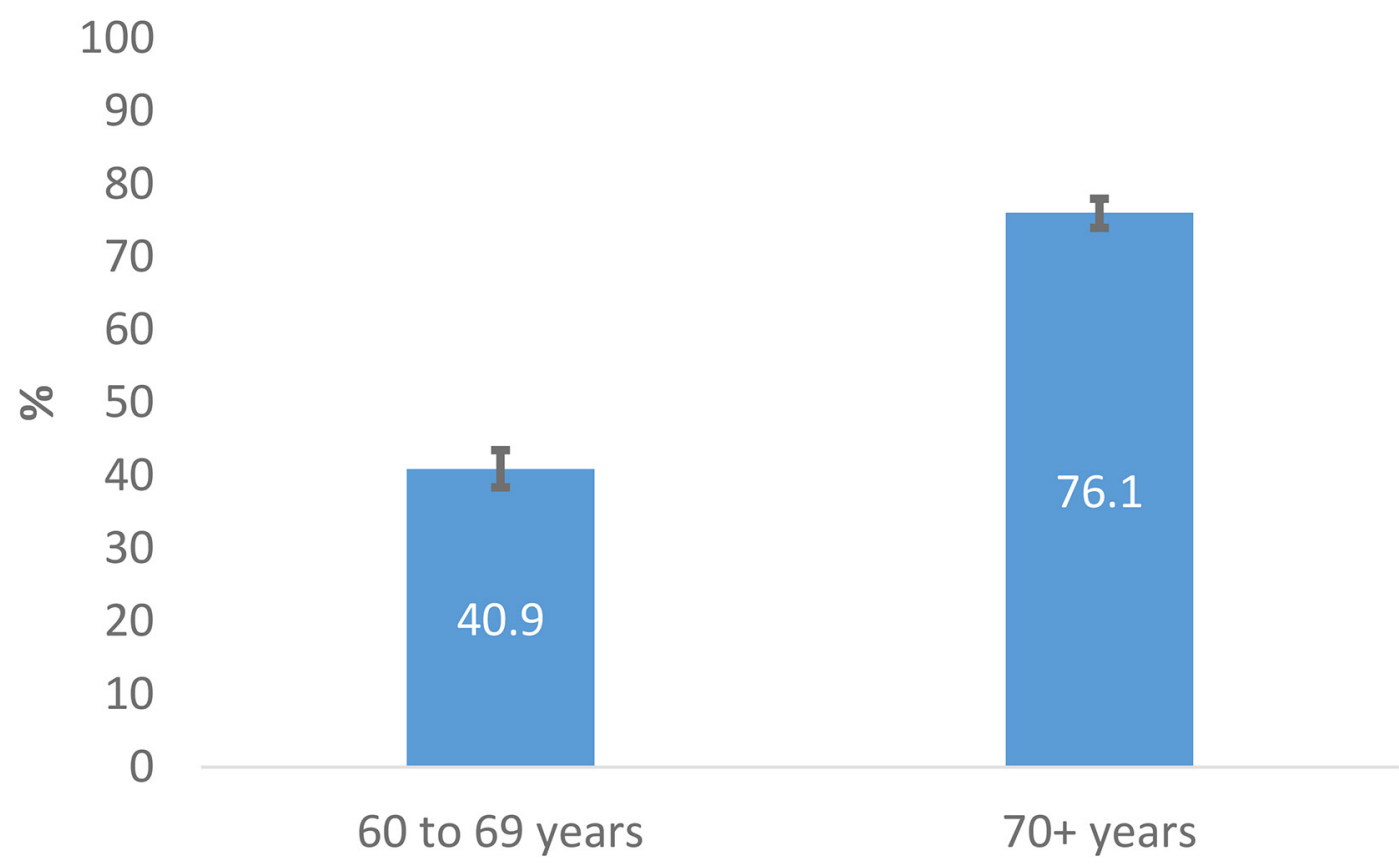

- As shown in Figure 2, there was a higher uptake of vaccination among adults aged over 60 who lived in Dublin city $(62 \%)$ or the surrounding areas $(61 \%)$ compared to those who lived in rural areas (56\%). 
Figure 2. Percentage of adults aged 60 and older in urban and rural areas who received the influenza vaccine in the two years prior to 2018/19

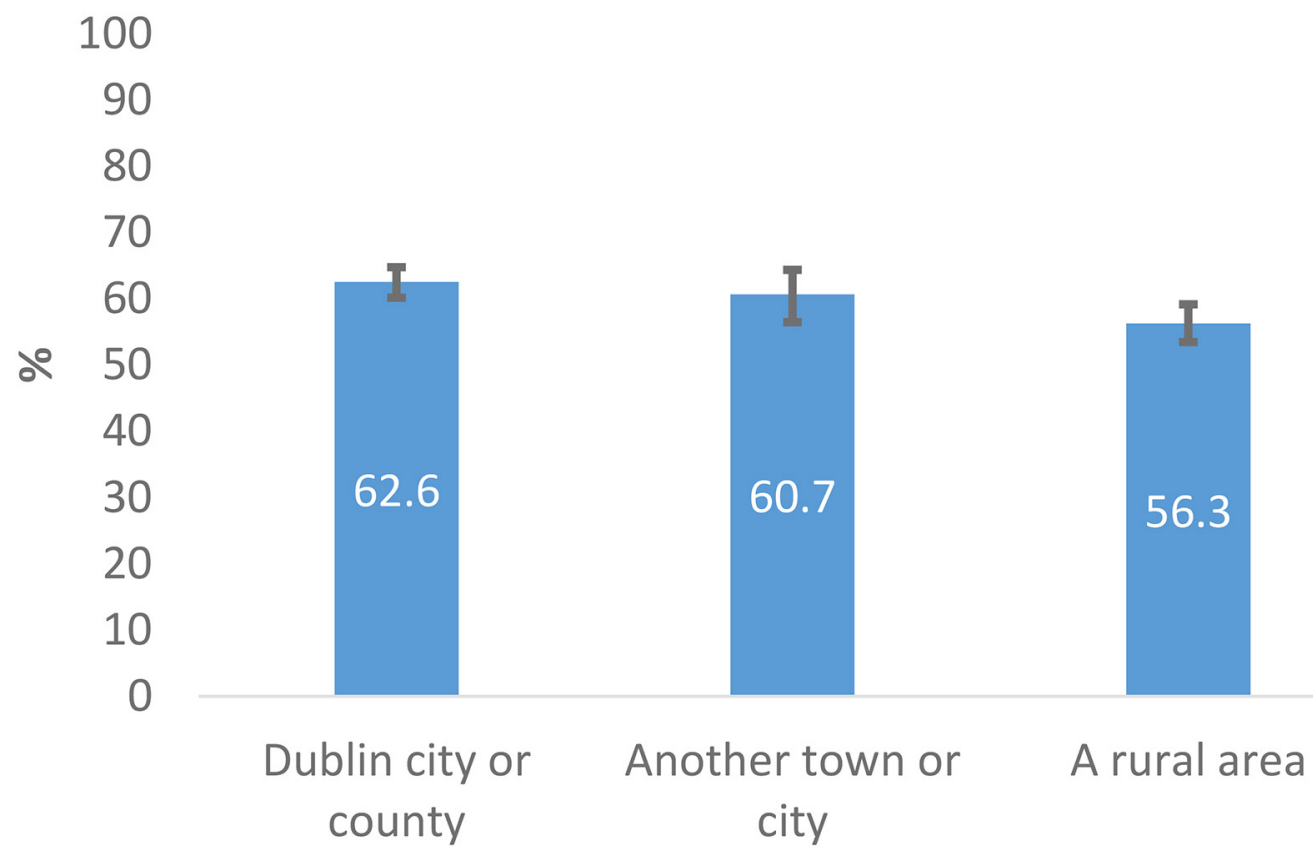

- With regard to the rate of vaccination by principal economic status (Figure 3), $69 \%$ of adults over 60 who were retired were vaccinated. This percentage was significantly higher than the rate of vaccination among individuals who were in employment (37\%) and among individuals who were either unemployed, permanently sick or disabled, looking after home or family, or in training (58\%).

Figure 3. Percentage of adults aged 60 and older who received the influenza vaccine in the two years prior to $2018 / 19$ by principal economic status

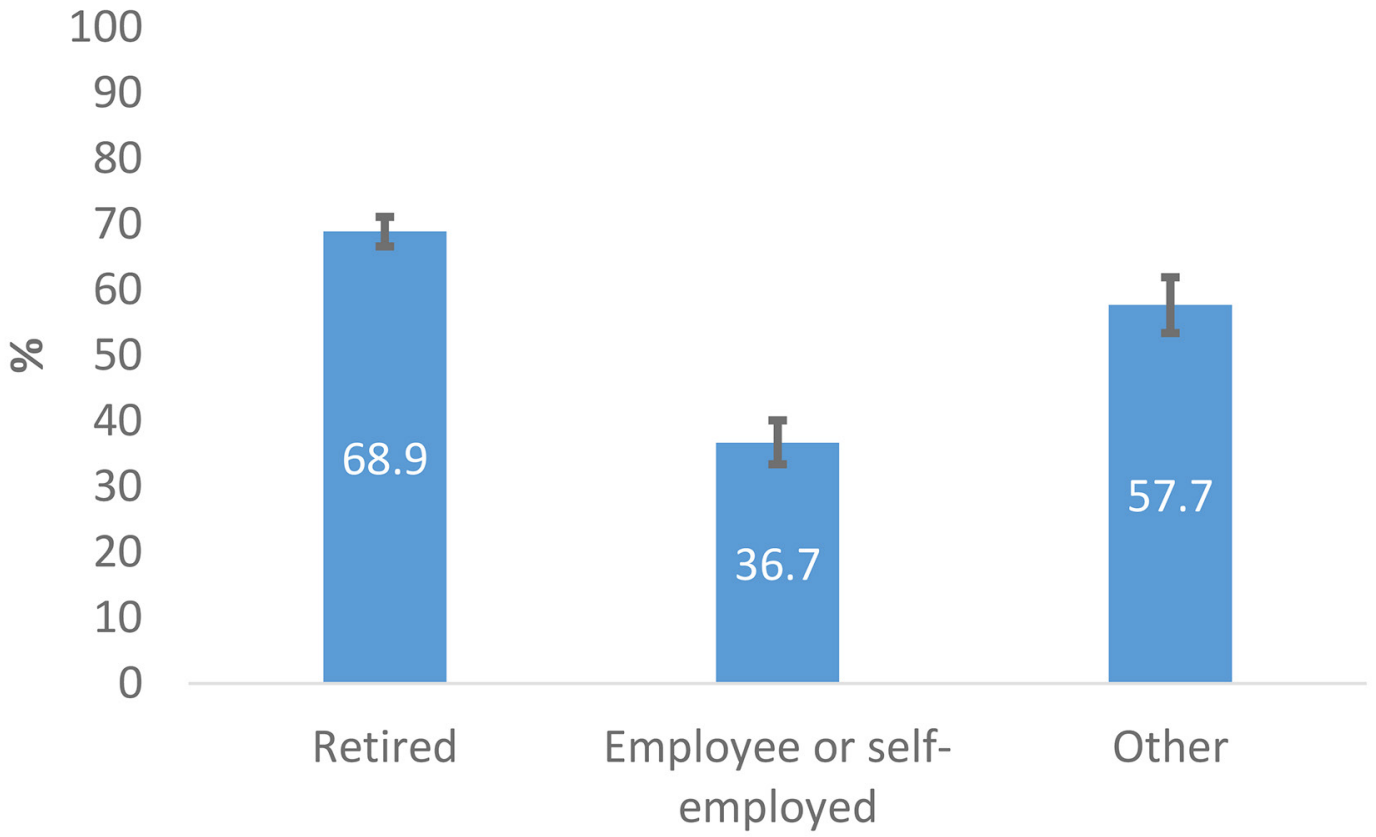


- Finally, as Figure 4 shows, the rates of vaccination were similar for adults over 60 who had a full medical card $(69 \%)$ or GP only medical card $(74 \%)$. Uptake among adults with private or no health insurance was significantly lower $(37 \%)^{1}$.

Figure 4. Percentage of adults aged 60 and older who received the influenza vaccine in the two years prior to $2018 / 19$ by medical insurance coverage

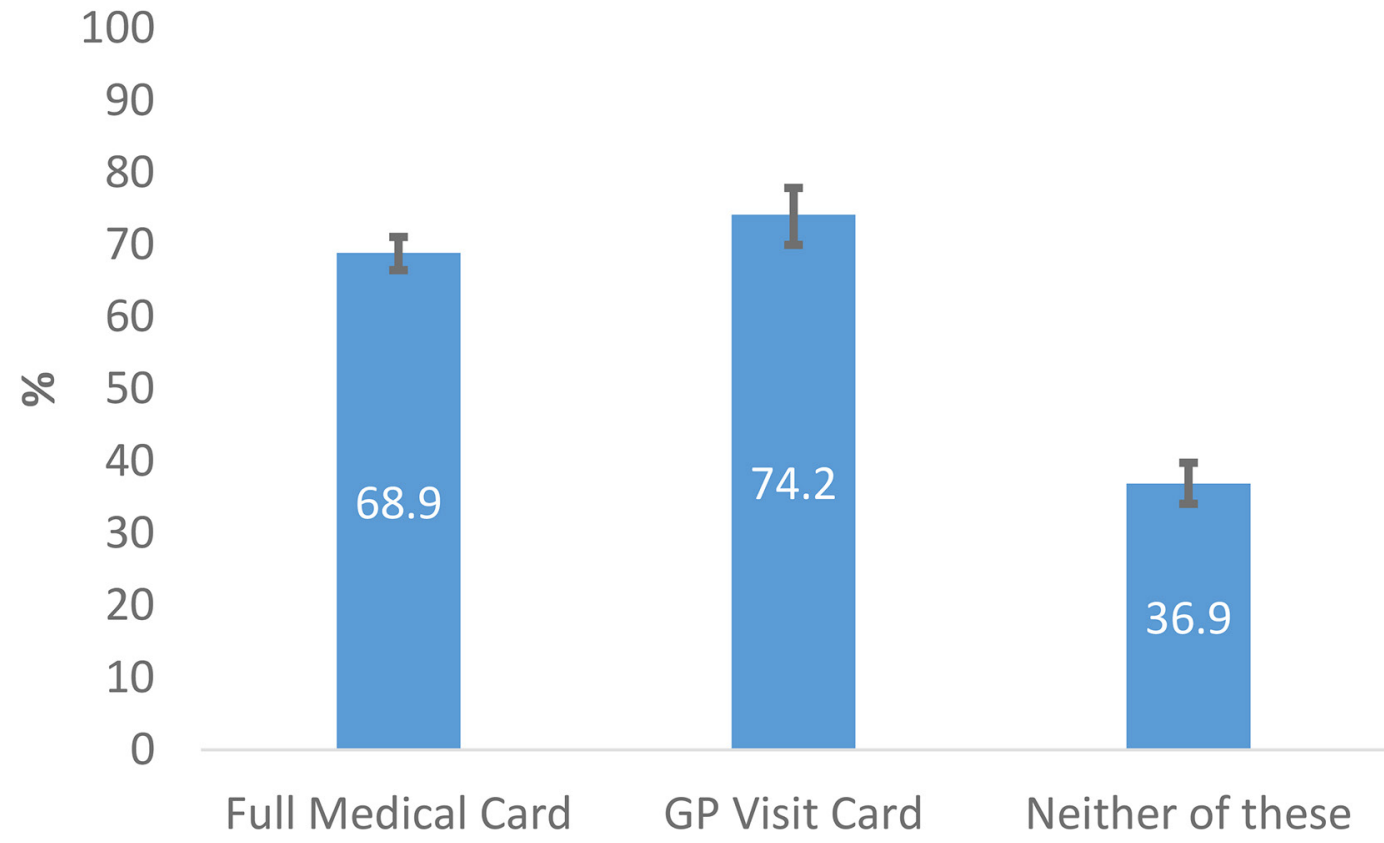

1 The influenza vaccination is free to all medical card and GP only medical card holders in the Republic of Ireland, as well as a number of at-risk groups, including all persons aged 65 years and older. Some private health insurers cover the cost of vaccination, otherwise a charge applies. 


\section{The effect of prolonged physical activity on vaccine efficacy}

\section{Nollaig Bourke}

The data reported in this section is based on up to to date review of the current peer reviewed a literature.

Vaccine efficacy in older adults can be a challenge due to the effects of ageing on the immune system. As people get older, the ability to mount robust antibody responses following vaccination declines. Older adults are therefore less likely to generate the long-term protection often required for full immunity against a pathogen. Vaccine efficacy is often evaluated based on the generation of these specific antibodies following vaccination. A key challenge is to try to boost these antibody responses in people with declining immune responses. This is particularly important as older adults are at a greater risk of hospitalisation or death if they contract the COVID-19 virus.

We conducted a literature review to assess the effect of physical activity on antibody responses to vaccination. We report the main findings below.

- Several randomised controlled trials (RCTs) have found that in older adults, prolonged aerobic exercise in the weeks and months prior to vaccination can help boost vaccine specific antibody responses $^{1-5}$. For example, aerobic exercise (treadmills, stair-steppers etc.) done 3 times per week for 10 months generated greater antibody responses following influenza vaccination ${ }^{1}$. Ten months of cardiovascular exercise also led to greater antibody responses 24 weeks after influenza vaccination ${ }^{3}$. Similarly, ten months of brisk walking 2-3 times per week conferred better antibody generation postvaccination in previous sedentary adults ${ }^{4}$. Practising Qigong and Taiji, traditional Asian martial arts, three times a week for 20 weeks also led to greater antibody responses to the influenza vaccine at 3, 6 and 20 weeks post vaccination ${ }^{5}$.

- There is less evidence from RCTs at this time to show that boosting physical activity immediately prior to vaccination can boost efficacy in older adults. The few studies done to date in this area showed that exercise immediately prior to vaccination had little effect on vaccine specific antibody responses. Therefore the positive effects of increasing physical activity on antibody generation post-vaccination seems to be dependent on the implementation of a regular regime of aerobic exercise in the weeks or months prior to vaccination.

- Cross-sectional studies have also shown that older adults who have higher levels of physical activity generate better antibody responses following vaccination ${ }^{6-9}$. Moderate and intense exercise lifestyle contributed towards post-influenza vaccine response antibody generation ${ }^{6}$. Similarly, regular and vigorous aerobic exercise had greater post-influenza vaccine antibody responses ${ }^{8}$. In addition, those termed "high-fit" older adults, based on a treadmill consumption test, had greater antibody responses to influenza vaccination? 
- Collectively, these cross-sectional studies strengthen the findings from the RCTs that prolonged moderate exercise prior to vaccination can enhance antibody responses post vaccination in older adults.

Based on this literature review, we recommend that adults aged over 60 should consistently incorporate some form of aerobic exercise (e.g. a walk brisk enough to leave one breathless) at least $2-3$ times per week in the weeks and months prior to vaccination. Evidence suggests that the more prolonged this exercise regime is, the better the immune responses will be to vaccination. 


\section{Levels of physical activity among TILDA participants who got the influenza vaccine between 2016 and 2019}

\section{Cillian McDowell}

The data reported in this section was collected by TILDA during the fifth wave of data collection in 2018/19.

In the fifth wave of data collected by TILDA in 2018/19, we asked participants how much time they spent being physically active in the last 7 days prior to their survey interview. We asked them how much time they engaged in moderate and vigorous physical activity. Moderate physical activities refer to activities that take moderate physical effort and make breathing somewhat harder than normal like brisk walking, carrying light loads, bicycling at a regular pace, or doubles tennis. Vigorous physical activities refer to activities that take hard physical effort and make breathing much harder than normal, like heavy lifting, digging, aerobics, or fast bicycling. We also asked TILDA participants how much time they spent walking.

Current guidelines recommend that adults aged over 60 engage in 150 minutes of moderate intensity activity, 75 minutes of vigorous intensity activity, or an equivalent combination, each week. Meeting these recommendations can be referred to as being "minimally physical active." Adults who report engaging in double this amount of activity (i.e., 300 minutes of moderate intensity activity, 150 minutes of vigorous intensity activity, or an equivalent combination, each week) are engaging in "health enhancing" physical activity.

- Among TILDA participants who got the influenza vaccine between 2016 and $2019,44 \%$ did less than the minimum recommended level of physical activity, $36 \%$ were minimally physically active, and just $20 \%$ engaged in "health enhancing" physical activity.

- As shown in Figure 5, women were significantly less likely to meet minimum recommended physical activity levels. $52 \%$ did not meet the minimum recommended level of physical activity compared to $35 \%$ of men. 
Figure 5. Percentage of adults aged 60 and older who received the influenza vaccine in the two years prior to $2018 / 19$ in each physical activity category by gender.

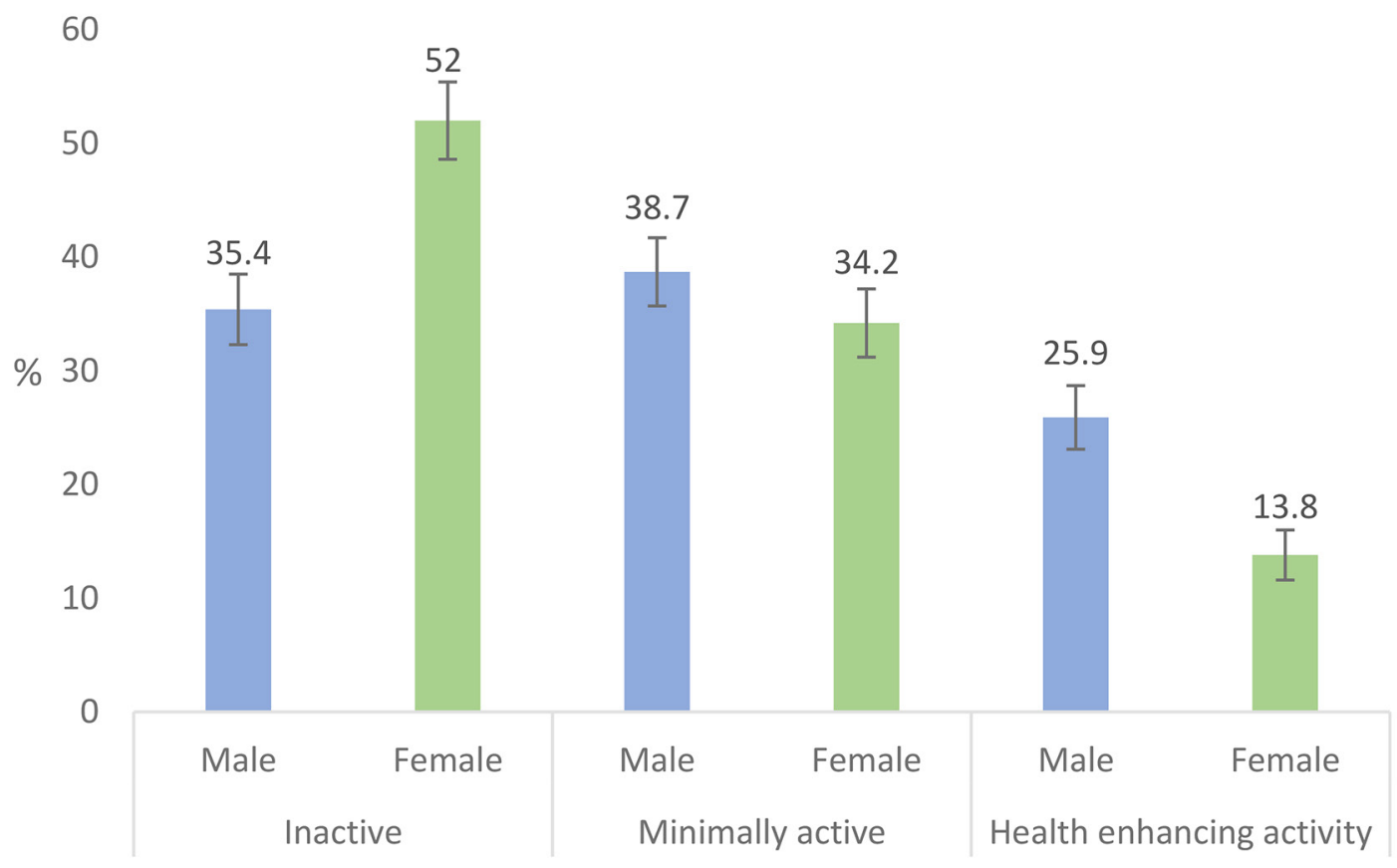

- As shown in Figure 6, 49\% of adults aged over 70+ who received the influenza vaccine did not meet minimum recommended physical activity levels, compared to $33 \%$ of adults aged $60-69$.

Figure 6. Percentage of adults aged 60 and older who received the influenza vaccine in the two years prior to $2018 / 19$ in each physical activity category by age.

60

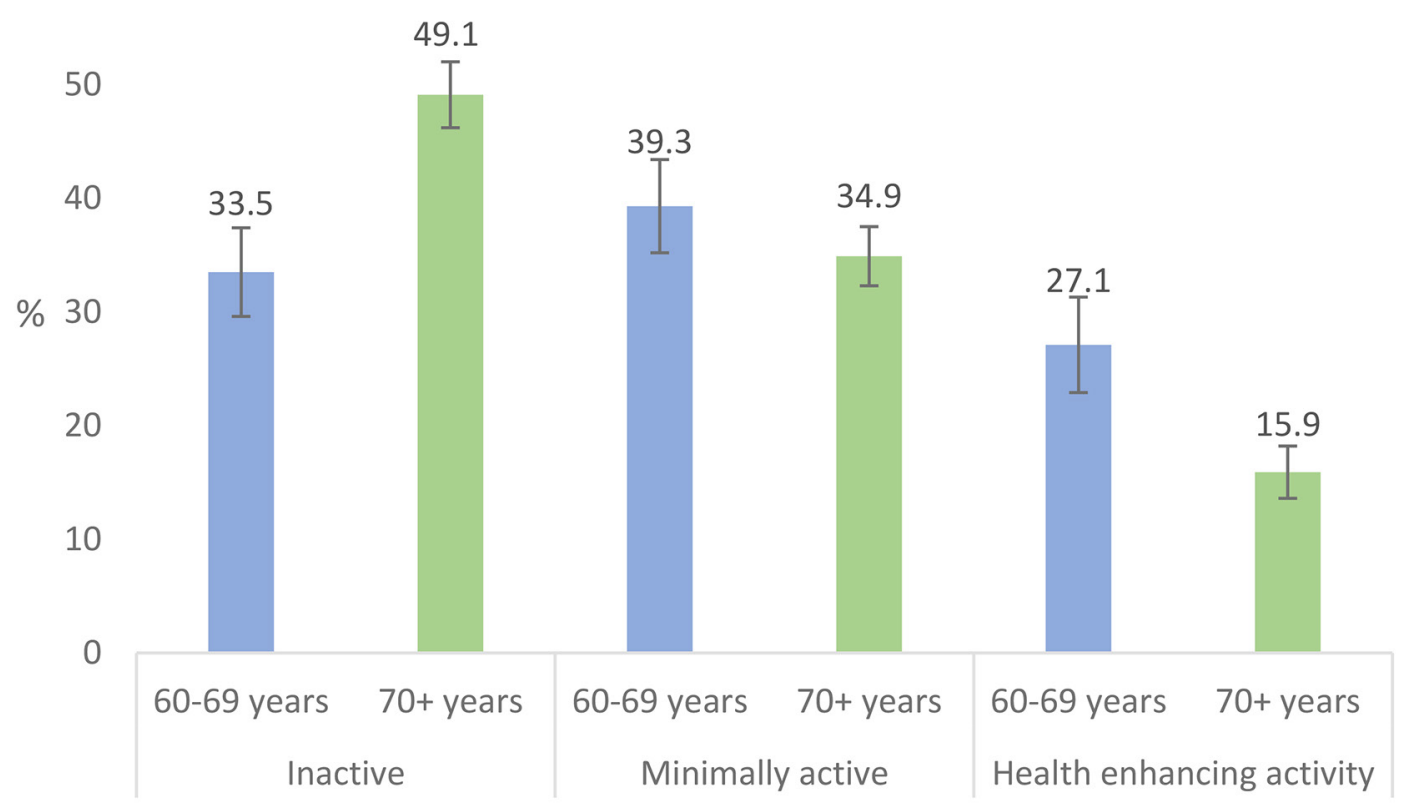


- Similarly, participants with a primary level of education or none were significantly less likely to meet minimum recommended physical activity levels (see Figure 7). 49\% did not meet the minimum recommended level of physical activity compared to $35 \%$ of those with a third or higher level of education.

Figure 7. Percentage of adults aged 60 and older who received the influenza vaccine in the two years prior to $2018 / 19$ in each physical activity category by education.

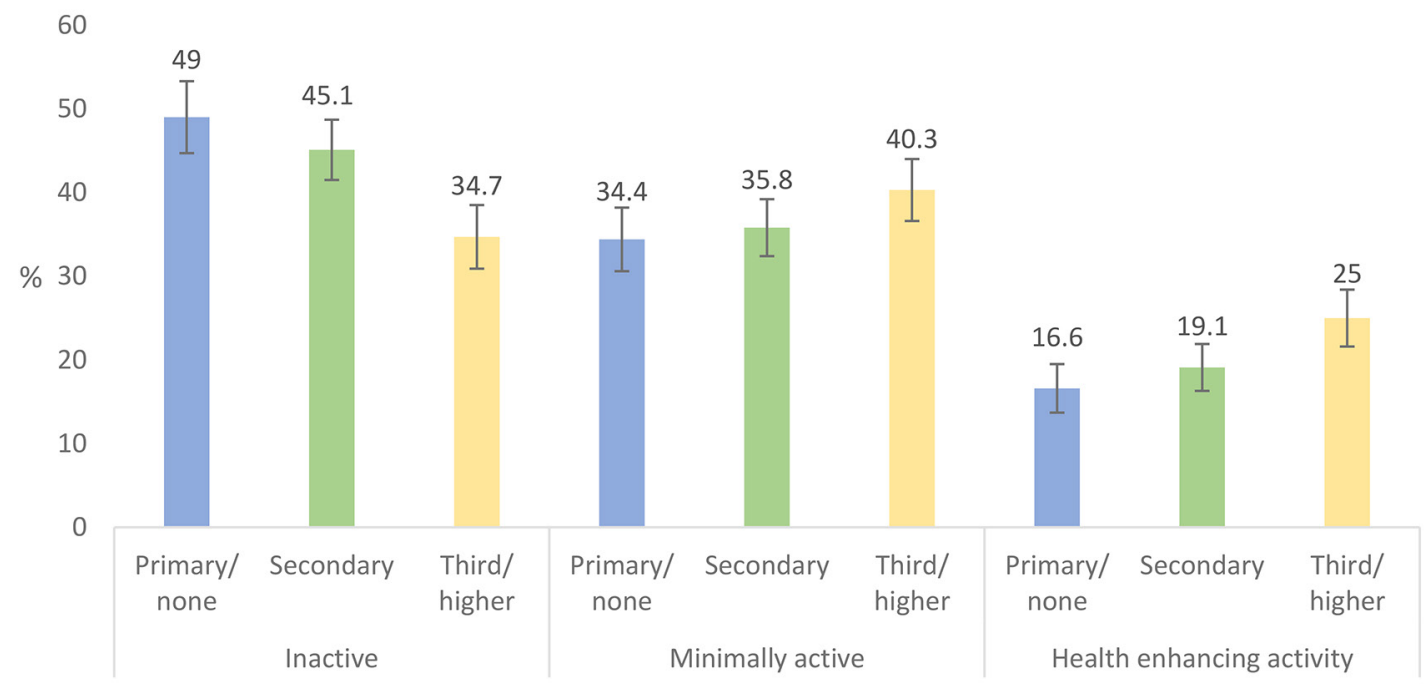

- Finally, as shown in Figure 8, participants who live in urban areas were significantly less likely to meet minimum recommended physical activity levels ( $46 \%$ and $35 \%$ compared to $31 \%$ of those living in rural areas).

Figure 8. Percentage of adults aged 60 and older who received the influenza vaccine in the two years prior to $2018 / 19$ in each physical activity category by household location.

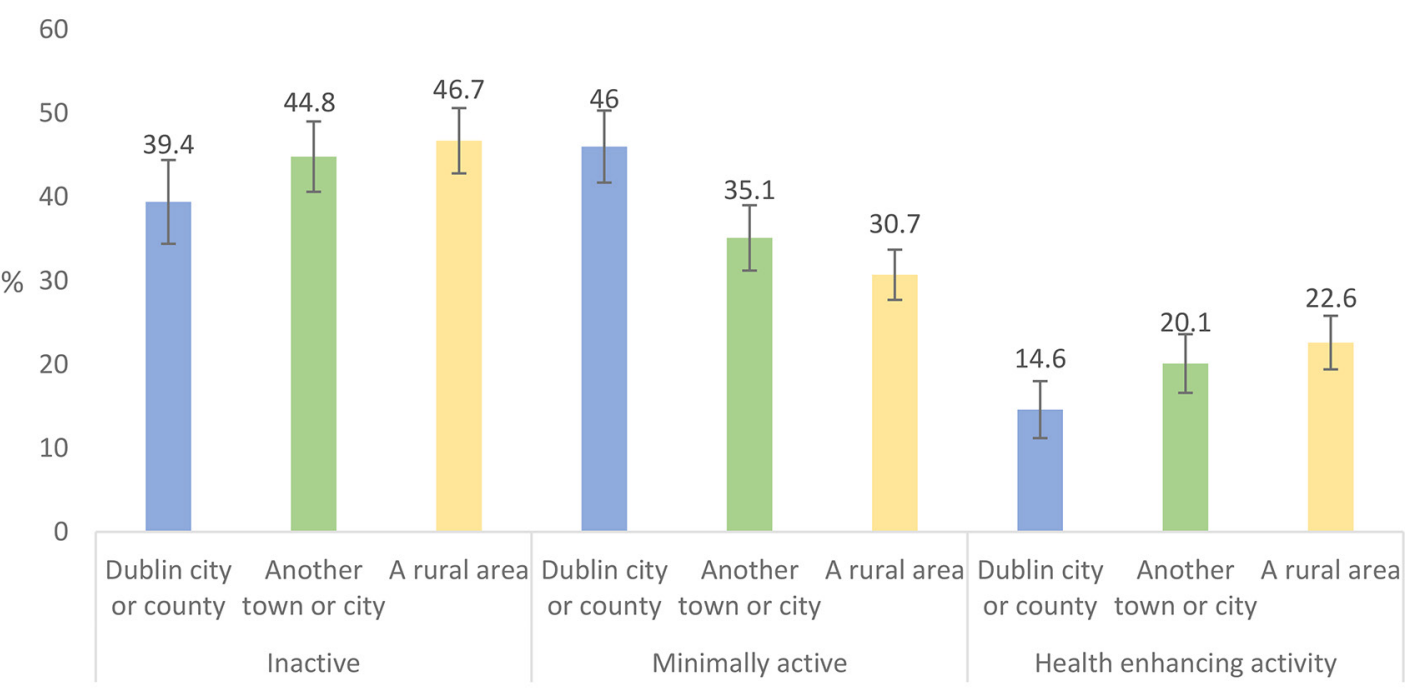




\section{TILDA participants' response to government's communication during the COVID-19 pandemic}

\section{Mark Ward}

The data reported in this section was collected as part of the TILDA COVID-19 self-completion questionnaire between June and October 2020.

\section{Sources used to get information on the COVID-19 pandemic}

It is important that information on vaccination for the COVID-19 virus is communicated via trusted news sources that adults aged over 60 in particular might access information on a vaccine. This data is of particular importance if it is indeed the case that priority will be given to people aged 65 and older in long term care facilities, closely followed by all adults aged 70 years and older. As part of the TILDA COVID-19 self-completion questionnaire administered to 3,600 community-dwelling adults aged 60 years and older between June and October 2020, participants were asked to name the media sources they used for news on COVID-19.

- As shown in Figure 9, 76\% of adults aged over 60 reported that they used Irish national broadcasters as a source of news during the COVID-19 pandemic.

- National radio stations (65\%) and national newspapers (43\%) were the next most often used news source.

- Older adults were more likely to use news media sources rather than official government sources with $7 \%$ using the Health Service Executive website and 6\% accessing information on the Department of Health website.

Figure 9. Percentage of adults aged 60 and older who used selected news sources during the COVID-19 pandemic

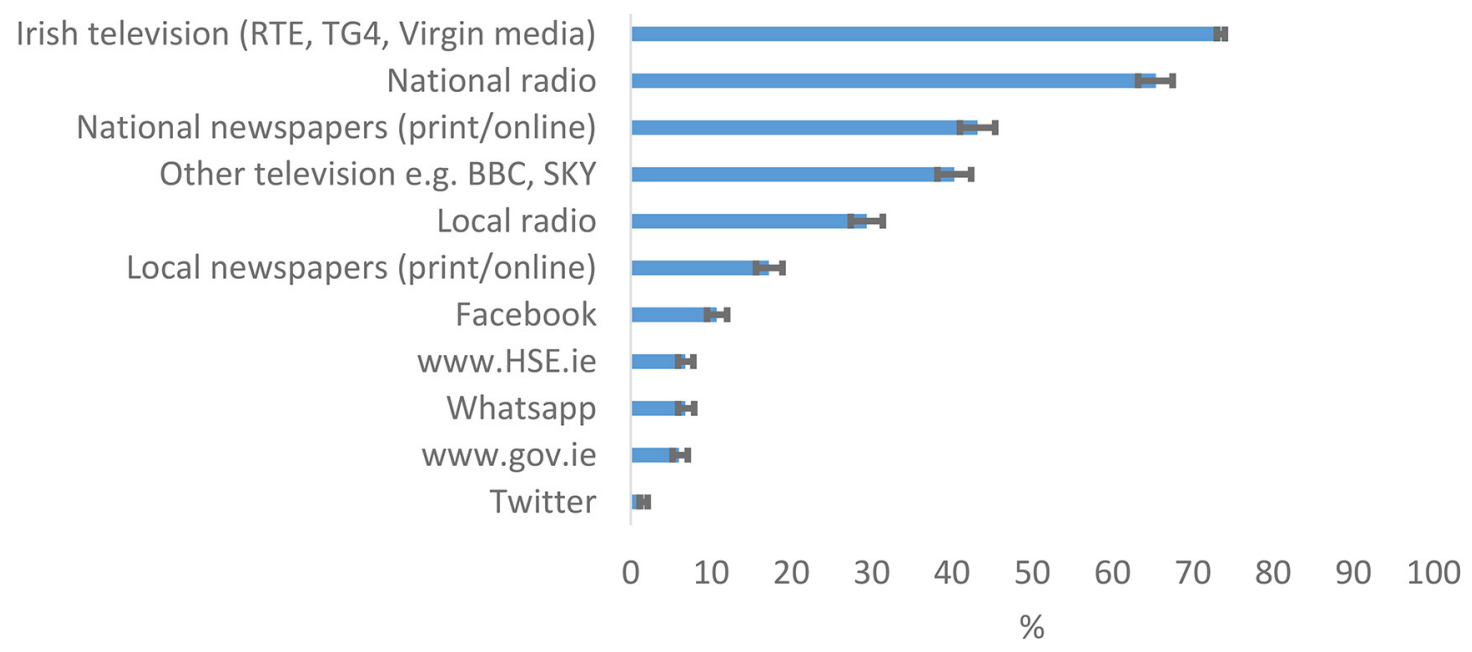


Trust in different sources of news about the COVID-19 pandemic

Participants who completed the COVID-19 self-completion questionnaire were also asked about their level of trust in a number of sources of news on COVID-19. Participants responded to this question on a scale of 1 to 10 , with 1 meaning they did not trust the source at all while 10 meant they trusted a source completely.

- The average level of trust in selected news sources (see details in Figure 10) among adults aged 60 and older during the COVID-19 pandemic was 7.7 out of a total of 10.

- The most trusted sources were the Irish television, the HSE, national radio, Government and local radio.

- Facebook and WhatsApp were the least trusted sources of information about the COVID-19 pandemic.

Figure 10. Level of trust on a scale selected news sources among adults aged 60 and older during the COVID-19 pandemic

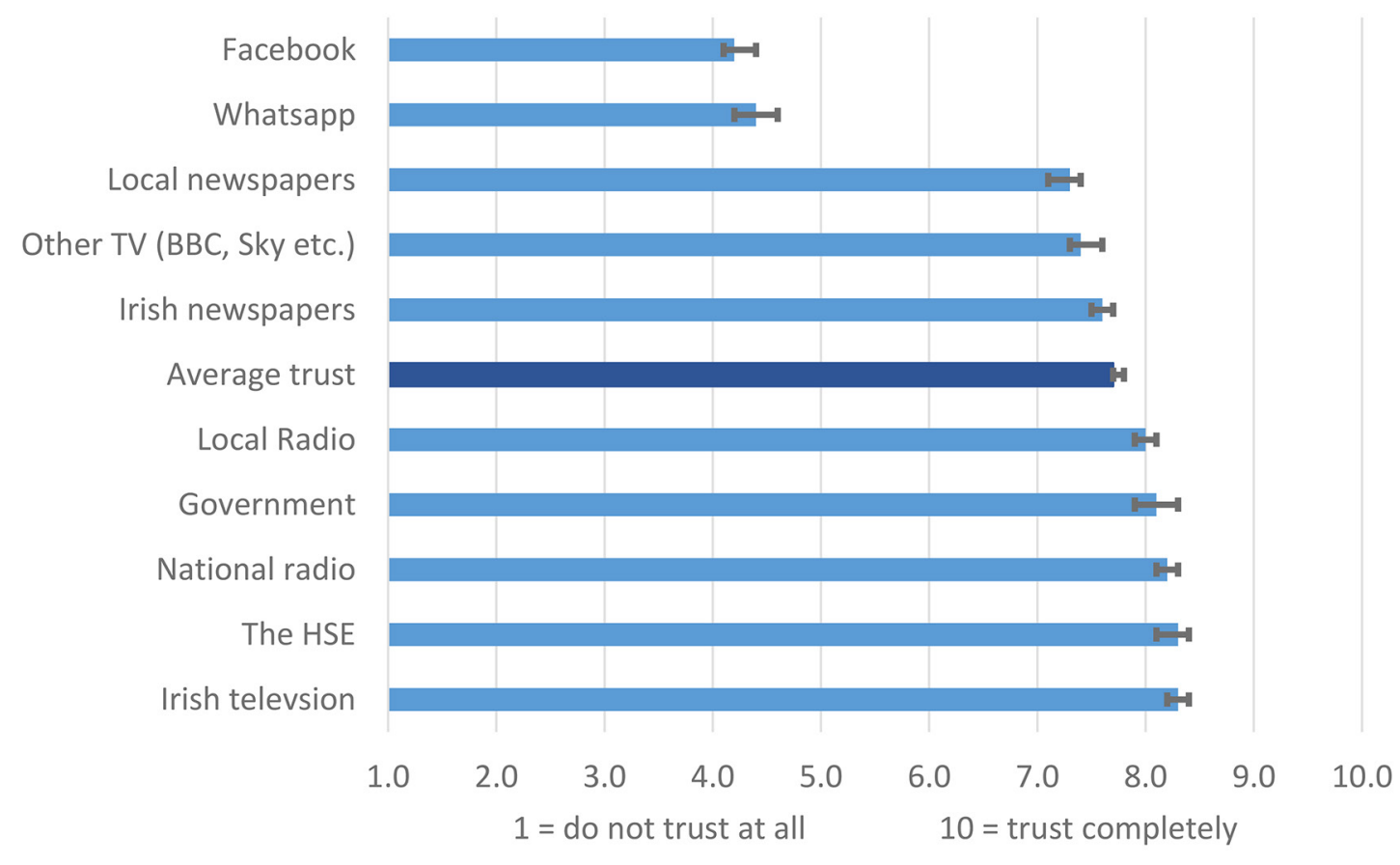




\section{Conclusion}

- This report first provided some insights of the patterns of vaccine uptake among community-dwelling adults aged 60 years and older in Ireland.

- 59 \% of adults aged over 60 received a influenza vaccination between 2016 and 2019.

- The groups that are less likely to receive vaccination are individuals aged 60-69 years; who live in rural areas; who are in employment; or who have a private or no health insurance.

- In addition, this brief informed about the positive effect of prolonged physical activity on antibody responses following vaccination. This is important information given that adults are less likely to mount robust antibody responses following vaccination and that $44 \%$ of adults aged over 60 in Ireland do less than the recommended level of physical activity.

- Adults aged 60 years and older should consistently incorporate some form of aerobic exercise (e.g. a walk brisk enough to leave one breathless) at least $2-3$ times per week in the weeks and months prior to vaccination.

- Campaigns should specifically target groups that are less likely to meet minimum recommended physical activity levels: that is, women; adults aged 75+; individuals with a primary level of education or none; and individuals who live in urban areas.

- Previous evidence has shown that the most effective physical activity messages among older adults include 1) highlighting the positive benefits of taking part in physical activity as opposed to the negative consequences of not taking part, 2) highlighting the short term social and mental health benefits of physical activity, and 3) avoiding technical language (e.g., "cardiovascular" vs "heart").

- It is important that information on a vaccine for the COVID-19 virus is communicated via trusted news sources that adults aged over 60 in particular might access information on a vaccine.

- TILDA data collected during the COVID-19 pandemic shows that adults aged 60 and older in Ireland are more likely to use Irish national broadcasters, national radio stations and national newspapers as a source of news.

- The most trusted sources are the Irish television, the HSE, national radio, Government and local radio. 


\section{References}

1. Kohut, M.L.; Arntson, B.A.; Lee, W.; Rozeboom, K.; Yoon, K.; Cunnick, J.E.; McElhaney, J. Moderate exercise improves antibody response to influenza immunization in older adults. Vaccine 2004, 22, 22982306.

2. Kohut, M.L.; Cooper, M.M.; Nickolaus, M.S.; Russell, D.R.; Cunnick, J.E. Exercise and psychosocial factors modulate immunity to influenza vaccine in elderly individuals. J. Gerontol. Ser. A Biol. Sci. Med. Sci. 2002, 57, M557-M562.

3. Vieira, V.; Keylock, K.T.; Lowder, T.; Zelkovich, W.; Dumich, S.; Colantuano, K.; Potter, K.; Leifheit, K.; Mcauley, E.; Woods, J.A. E_ects of exercise training on the immune response to influenza vaccination in older adults: A randomized controlled trial. Brain Behav. Immun. 2008, 22, 26.

4. Woods, J.A.; Keylock, K.T.; Lowder, T.; Vieira, V.J.; Zelkovich, W.; Dumich, S.; Colantuano, K.; Lyons, K.; Leifheit, K.; Cook, M.; et al. Cardiovascular exercise training extends influenza vaccine seroprotection in sedentary older adults: The immune function intervention trial. J. Am. Geriatr. Soc. 2009, 57, 2183-2191.

5. Yang, Y.; Verkuilen, J.; Rosengren, K.S.; Mariani, R.A.; Reed, M.; Grubisich, S.A.; Woods, J.A. E_ects of a Taiji and Qigong intervention on the antibody response to influenza vaccine in older adults. Am. J. Chin. Med. 2007, 35, 597-607.

6. De Araújo, A.L.; Silva, L.C.; Fernandes, J.R.; De Sousa Toledo Matias, M.; Boas, L.S.V.; Machado, C.M.; Garcezleme, L.E.; Benard, G. Elderly men with moderate and intense training lifestyle present sustained higher antibody responses to influenza vaccine. Age 2015, 37, 105.

7. Keylock, K.T.; Lowder, T.W.; Leifheit, K.; Cook, M.D.; Mariani, R.A.; Ross, K.M.; Kim, K.; Chapmannovakofski, K.; Mcauley, E.; Woods, J.A. Higher antibody, but not cell-mediated, responses to vaccination in high physically fit elderly. J. Appl. Physiol. 2007, 102, 1090-1098

8. Kohut, M.L.; Cooper, M.M.; Nickolaus, M.S.; Russell, D.R.; Cunnick, J.E. Exercise and psychosocial factors modulate immunity to influenza vaccine in elderly individuals. J. Gerontol. Ser. A Biol. Sci. Med. Sci. 2002, 57, M557-M562.

9. Schuler, P.B.; Leblanc, P.A.; Marzilli, T.S. E_ect of physical activity on the production of specific antibody in response to the 1998-99 influenza virus vaccine in older adults. J. Sports Med. Phys. Fit. 2003, 43, 404-408. 